\title{
Relación entre clima organizacional y violencia laboral en funcionarios de una universidad pública
}

\author{
Relationship between organizational climate and workplace violence in public university officials \\ Anyi Katherine Pantoja-Pantoja' orcid.org/0000-0002-4922-9020 \\ Adriana Patricia Navarrete-Acosta' ${ }^{1}$ orcid.org/0000-0003-3099-3755 \\ Christian Alexander Zambrano-Guerrero',2 orcid.org/0000-0001-8482-0345 \\ Sonia Maritza Matabanchoy-Tulcan 1,3* orcid.org/0000-0002-3681-8440
}

1 Programa Psicología, Universidad de Nariño. Pasto, Colombia

2 Grupo de Investigación Psicología y Salud, Universidad de Nariño. Pasto, Colombia

3 Grupo de Investigación CONEPSI, Universidad de Nariño. Pasto, Colombia

\section{Resumen}

Introducción: Introducción: El clima laboral se relaciona con el acoso psicológico en el trabajo. No obstante, son escasos los estudios encontrados sobre la relación entre clima y violencia laboral en el contexto universitario. Objetivo: Analizar la relación entre el clima y la violencia laboral en funcionarios de una universidad pública. Materiales y métodos: Estudio descriptivo correlacional con diseño transversal, con la participación de 410 funcionarios. Se utilizó el Cuestionario de Clima Laboral del Departamento Administrativo de la Función Pública de Colombia y el Inventario de Violencia y Acoso Psicológico en el Trabajo. Resultados: Se encontró que existe una relación negativa entre el clima y la violencia laboral $(r=-0,441, p<0,005)$, en esta se resaltan estrechas asociaciones entre la dimensión obstáculos al desempeño laboral, y estilo de liderazgo, administración del talento humano y clima general. Conclusiones: La presencia de violencia laboral se relaciona con bajos niveles de favorabilidad de clima laboral y viceversa. Los comportamientos característicos de obstáculos al desempeño laboral y los referentes a "manipulación para inducir al castigo" son los que aportan en mayor medida a esta desfavorabilidad. Este tipo de violencia, sin una debida intervención puede llegar a convertirse en acoso psicológico en el trabajo.

Palabras clave: Violencia laboral; relaciones laborales; institución; trabajadores. (Fuente: DeCS, Bireme).

\begin{abstract}
Introduction: Work environment isaffected by psychological harassment in the workplace. However, there are few studies on the relationship between workplace climate and violence in the university context. Objective: To analyze the relationship between workplace climate and violence in public university employees. Materials and Methods: A descriptive correlational study with a crosssectional design was conducted with 410 workers. The Work Climate Questionnaire of the Administrative Department of Public Service of Colombia and the Inventory of Violence and Psychological Harassment at Work were used. Results: It was found that there is a negative relationship between climate and violence in the workplace $(r=-0.441, p<0.005)$, in which two close associations are highlighted: (i) the dimension of work performance obstacles and the leadership style; and (ii) human talent management and general climate. Conclusions: The presence of workplace violence is related to low levels of a favorable work environment and vice versa. The characteristic behaviors of the dimension world performance obstacles and those related to the variable manipulation to induce punishment are the ones that contribute the most to this unfavorable association. This type of violence, without proper intervention, can become psychological harassment at work.
\end{abstract}

Keywords: Labor violence, Labor relations, Organization; workers. (Source: DeCS, Bireme)

\footnotetext{
*Autor de correspondencia: 


\section{Introducción}

Toda organización requiere que su equipo de trabajo se articule con los objetivos y metas que orientan su adecuado funcionamiento y desarrollo(1). A partir de la revisión de la literatura científica, se ha observado que son escasos los estudios sobre la relación entre el clima organizacional y violencia laboral; sin embargo, se destaca que en las dinámicas de una organización, el clima laboral se relaciona teóricamente con la presencia o no de una forma de violencia laboral denominada acoso psicológico en el trabajo(2-5).

\section{Clima organizacional}

El clima organizacional presenta abordajes teóricos que conducen a diversas interpretaciones $y$ explicaciones sobre los factores y aspectos que se relacionan en su construcción. Los autores coinciden en que el clima se refiere a las percepciones, compartidas o no, que tienen los individuos en cuanto a la organización en sus diferentes aspectos, como: el ambiente físico y las relaciones interpersonales, entre otras (6,7). En coherencia con la multidimensionalidad del concepto de clima laboral, existen diversos componentes evaluados por los diferentes instrumentos ${ }^{(8)}$, los más comunes son: toma de decisiones, claridad organizacional, liderazgo, interacción social, motivación, sistema de recompensas e incentivos, apertura organizacional y supervisión(8).

El estudio del clima organizacional en Colombia propone un concepto integrado que permite dar cuenta del comportamiento de los colaboradores a partir de la influencia de las políticas, prácticas administrativas, la tecnología y los procesos de toma de decisiones(8). Asimismo, se afirma que este está determinado por procesos psicológicos o variables internas que afectan el comportamiento del colaborador ${ }^{(9)}$, otorgándole un carácter subjetivo que retoma las percepciones y los significados que el hombre construye en un determinado ambiente. En el ámbito educativo, por ejemplo, intervienen variables propias de este contexto, entre ellas la académica, la socio afectiva, la administrativa y la ética(10).

El Departamento Administrativo de la Función Pública (DAFP), entidad técnica, estratégica y transversal del Gobierno colombiano, ha elaborado una propuesta para la intervención del clima organizacional, también denominado clima laboral, en la cual se tienen en cuenta siete dimensiones(11). Esta entidad define el clima como "el conjunto de percepciones y sentimientos compartidos que los funcionarios desarrollan en relación con las características de su entidad, tales como políticas, prácticas y procedimientos formales e informales y las condiciones de la misma"(11). Esta definición se acoge en los procesos de evaluación y conceptualización del clima laboral, considerando la interacción entre el colaborador y el entorno de la institución pública de educación superior donde se realizó la investigación. La importancia del conocimiento y la gestión del clima laboral en las diferentes dependencias de la institución, se basa en la influencia que este ejerce sobre el comportamiento de los colaboradores(12).

De los instrumentos revisados, se considera que el Cuestionario de Clima Laboral del Departamento Administrativo de la Función Pública (DAFP), validado en Colombia y dirigido específicamente a la evaluación del clima en funcionarios públicos(11), aborda gran parte de las características comunes de este constructo.

\section{Violencia laboral}

La violencia laboral está asociada con hechos, acciones $o$ incidentes violentos, desde las manifestaciones abiertas de violencia física o verbal hasta las formas más sutiles(13). Muchos trabajadores son sometidos a maltrato, acoso sexual, amenazas, manifestaciones de incivismo, intimidación y otras formas de violencia(14). Esta variable se define como toda acción o incidente no razonable en la cual un trabajador es agredido, amenazado, humillado o lesionado en el ejercicio de su actividad profesional o como consecuencia directa de la misma(15).

Las consecuencias psicológicas por la exposición a la violencia laboral van desde la disminución de la satisfacción laboral y el aumento del absentismo, hasta el deterioro del bienestar psicológico y el desarrollo de psicopatologías(16). Constituye uno de los estresores más importantes en el ámbito del trabajo, generando graves consecuencias a nivel individual y organizacional(17). Esta suele presentarse tanto en sentido vertical (ascendente o descendente) como entre pares, la cual puede ejercerse por acción u omisión, afectando la salud y el bienestar de las personas que trabajan, y 
configurando una violación a los derechos humanos y laborales(18).

La California Division of Occupational Healthand Safety (Cal/OSHA) ofrece una clasificación de tres tipos de violencia, considerando las personas implicadas y el tipo de relación que existe entre ellas. En la violencia tipo I, quien ejerce acciones violentas no tiene ninguna relación legítima de trato con la víctima, a diferencia de la violencia tipo II, la cual se presenta al ofrecer o recibir algún servicio, por su parte la violencia tipo III, es ejercida por personas con implicación laboral directa o indirecta, actual o pasada (19). Para la presente investigación se tuvo en cuenta el último tipo de violencia, escogida en coherencia con el objetivo del estudio.

En el contexto universitario, se presentan algunas condiciones organizacionales e individuales como el clima, la cultura y el estilo de supervisión, que predisponen el desarrollo de violencia laboral(20). A nivel individual se encuentran las características de la personalidad, tanto en la víctima como en el perpetuador(20). Específicamente en la institución donde se realizó el presente estudio, se tiene como antecedente una investigación sobre violencia basada en género, de la cual se derivó la categoría emergente violencia laboral(21), hecho que despierta el interés de realizar una profundización sobre dicha variable en este contexto.

\section{Acoso psicológico en el trabajo}

El acoso psicológico en el trabajo es un elemento que hace parte de la violencia laboral, este se distingue por ser un conjunto de conductas premeditadas, continuadas, sostenidas en el tiempo y encaminadas a la eliminación de la víctima(18).

Leymann(22) proporcionó la primera definición técnica de acoso psicológico, teniendo en cuenta aspectos como el tiempo (seis meses o más) y la frecuencia (casi todos los días) en la presentación de acciones hostiles, por una o varias personas hacia una tercera. La intimidación o el acoso en el lugar de trabajo implican hostigar, ofender, excluir socialmente a alguien o afectar negativamente su trabajo(23). Este se clasifica de acuerdo a su direccionalidad, vertical (el acosador está en posición de poder superior a la de su víctima) de abajo hacia arriba (los acosadores se unen contra alguien que tiene una posición superior), horizontal (se trata de acoso entre iguales) y mixto (cuando varias de estas situaciones se encuentran presentes a la vez en un acto de acoso)(24).

Estas definiciones coinciden en gran medida con la legislación Colombia, en la cual se define el acoso psicológico en el trabajo como "la conducta de carácter persistente y demostrable, que se da con la intención de generar miedo y causar perjuicio laboral, generar desmotivación en el trabajo, o inducir la renuncia del mismo" (25).

A partir de investigaciones realizadas en el contexto colombiano se encontró que un 7,8\% de la población es víctima de acoso psicológico en el trabajo(26). Asimismo, el Ministerio de la Protección Social realizó un estudio en el que se reportó un 19,8\% de acoso psicológico. Entre los sectores económicos más vulnerables se encontró el de educación con 19,7\%(27). Debido a su prevalencia se considera que éste es un tema importante que exige atención inmediata.

\section{Relación entre clima organizacional y violencia laboral}

Son escasos los estudios sobre la relación entre el clima organizacional y la violencia laboral. Las investigaciones encontradas ${ }^{(2-5,28,29)}$ se han focalizado en el análisis de esta relación respecto a una forma de violencia, denominada acoso psicológico en el trabajo. En este sentido, se afirma que la existencia de hostigamiento laboral acarrea repercusiones negativas en la persona hostigada, genera un clima laboral negativo que refleja sus consecuencias en la producción organizacional(4). De esta manera, el clima laboral se puede estudiar como causa, efecto o condición intermediaria del acoso, ya que presenta una estrecha relación teórica bidireccional con este constructo, junto a diferentes situaciones en los puestos de trabajo(3).

En el caso de los estudios empíricos, se encuentra que la presencia de prácticas de acoso resulta contingente, tanto al tipo de clima que predomina en dicho entorno, como a los procesos de cambio organizacional que se dan en el mismo(28,29). En este sentido, se puede mencionar que un clima laboral deteriorado, es el contexto ideal para la presencia de violencia laboral y el desarrollo de acoso psicológico en el trabajo(29).

Analizar el clima organizacional respecto a la violencia laboral, puede aportar al entendimiento de 
las dinámicas propias del contexto educativo, ya que el abordaje de esta relación se ha venido haciendo específicamente en correspondencia al acoso, así el propósito de la presente investigación fue analizar la relación entre el clima y la violencia laboral en los funcionarios de una universidad pública.

\section{Materiales y métodos}

Se estableció un estudio cuantitativo de tipo descriptivo correlacional con diseño transversal(30). La población estuvo conformada por 1501 funcionarios de una universidad pública de San Juan de Pasto, mediante un muestreo estratificado con una afijación proporcional, se obtuvo una muestra de 410 participantes entre administrativos $(39,7 \%)$, directivos $(4,8 \%)$, docentes $(36,7 \%)$ y personal de apoyo $(18,8 \%)$. Con respecto al nivel de estudio alcanzado, el 13,24\% cursaron hasta básica segundaria, el 11\% son técnicos y tecnólogos, el 23\% son profesionales, el $10 \%$ han cursado maestría y el $47 \%$ han alcanzado el título de doctorado.

Para la recolección de información, se utilizó el Cuestionario de Clima Laboral del DAFP, que evalúa siete dimensiones: orientación organizacional (00), administración del talento humano (ATH), estilo de dirección o liderazgo (ED), comunicación e integración (CI), trabajo en grupo (TG), capacidad profesional (CP) y medio ambiente físico (MAF) ${ }^{(11) .}$

Para medir violencia laboral en el trabajo se aplicó el Inventario de Violencia y Acoso Psicológico en el Trabajo (IVAPT) validado para Colombia, con el fin de conocer la presencia e intensidad de la violencia y la existencia de mobbing. Este instrumento mide tres componentes, manipulación para inducir al castigo (MIC), daños a la imagen pública (DIP) y obstáculos al desempeño laboral (ODL)(26).

Para el desarrollo del estudio, inicialmente se contó con el aval del Consejo de Administración de la Universidad, a continuación, se realizó una fase de sensibilización e información con los participantes. Los instrumentos de medición se sometieron a validación de contenido, con la participación de cinco jueces profesionales expertos en el área de medición y evaluación y en el campo de la psicología organizacional, quienes evaluaron pertinencia, coherencia y claridad de los ítems, posteriormente se hicieron los ajustes y se diseñó el formato digital.

Una vez se obtuvieron los datos, se procedió a determinar la confiabilidad de los instrumentos a través de la consistencia interna. El Cuestionario de Clima Laboral del Departamento Administrativo de la Función Pública obtuvo un Alfa de Cronbach de 0,932 , se realizó un análisis exploratorio por cuadrados no ponderados (ULS), considerando que las distribuciones de las variables resultaron extremas(31), mediante el método de rotación varimax, que corresponde a un método de rotación ortogonal, en el cual se asumen la independencia de los factores(32),obteniendo una solución de cinco factores con altos índices de confiabilidad para cada uno de ellos (ED $(0,87)$ CP $(0,87)$ ATH $(0,83)$ TG $(0,76)$ MAF $(0,84))$.Por su parte, el Inventario de Violencia y Acoso Psicológico en el Trabajo IVAPT obtuvo una confiabilidad de 0,929 . (tabla 1 )

El análisis de resultados se realizó con el software SPSS versión 23, las variables clima organizacional, violencia laboral y acoso psicológico en el trabajo fueron evaluadas por separado, se calculó la distribución de las variables, se identificó una ausencia de normalidad en los datos y finalmente se utilizó el coeficiente de Spearman.

\section{Consideraciones éticas}

De acuerdo con la normatividad colombiana establecida en la Resolución 8430 de 1993 del Ministerio de Salud(33), el presente estudio se clasifica como una investigación sin riesgo. El estudio contó con el aval del Comité de Ética de la Universidad y la autorización al Consejo de Administración, que permitió continuar con la fase de sensibilización e información, previa a la aplicación de los instrumentos. Se obtuvo el consentimiento informado por escrito que garantiza el anonimato, la confidencialidad y la participación libre e informada en el proceso de investigación. 
Tabla 1. Descripción de la carga factorial de los ítems del instrumento de Clima Organizacional del Departamento Administrativo de la Función Pública (DAPF)

\begin{tabular}{|c|c|c|}
\hline Características Evaluadas en cada Dimensión & $\begin{array}{c}\text { Carga } \\
\text { factorial }\end{array}$ & Alpha de Cronbach \\
\hline \multicolumn{3}{|l|}{ Estilo de dirección } \\
\hline Mi jefe tiene en cuenta las sugerencias que le formulo & 0,795 & \\
\hline Mi jefe es coherente en sus argumentos y planteamientos. & 0,785 & \\
\hline $\begin{array}{l}\text { Mi jefe me motiva y reconoce mi trabajo } \\
\text { Mi jefe asigna el trabajo teniendo en cuenta los conocimiento y habilidades de sus colabora } \\
\text { Mi jefe tiene los conocimientos y las destrezas para dirigir el área } \\
\text { El trato que recibo de mi jefe es respetuoso } \\
\text { Mi jefe me tiene en cuenta para desarrollar trabajos importantes } \\
\text { En mi área, el jefe asigna las cargas de trabajo de forma equitativa }\end{array}$ & $\begin{array}{l}0,747 \\
0,675 \\
0,671 \\
0,669 \\
0,652 \\
0,624\end{array}$ & 0,87 \\
\hline Las decisiones que toman los grupos de trabajo de mi área son apoyadas por el jefe. & 0,536 & \\
\hline Recibo de mi jefe retroalimentación tanto de aspectos positivos como de aspectos negativos de mi trabajo & 0,301 & \\
\hline \multicolumn{3}{|l|}{ Capacidad Profesional } \\
\hline En mi trabajo hago una buena utilización de mis conocimientos & 0,726 & \\
\hline Tengo claridad de por qué realizo mis trabajos & 0,711 & \\
\hline Me encuentro satisfecho con el trabajo que realizo. & 0,664 & \\
\hline Tengo las habilidades requeridas para realizar mi trabajo. & 0,657 & \\
\hline Estoy dispuesto a hacer un esfuerzo extra cuando sea necesario por el bien de la entidad & 0,645 & \\
\hline Entiendo claramente mi papel dentro del área de trabajo a la que pertenezco. & 0,615 & \\
\hline Supero las expectativas que mi jefe tiene sobre mi desempeño. & 0,544 & 0,87 \\
\hline Puedo manejar adecuadamente mi carga de trabajo & 0,536 & \\
\hline Siento que estoy ubicado en el cargo que desempeño, de acuerdo con mis conocimientos y habilidades. & 0,507 & \\
\hline Considero que mis funciones están claramente determinadas. & 0,49 & \\
\hline Me siento motivado para trabajar por el mejoramiento de los procesos de trabajo. & 0,393 & \\
\hline Los objetivos de mi entidad son claros & 0,329 & \\
\hline Conozco la misión y la visión de mi entidad. & 0,321 & \\
\hline Cuando surge un problema, sé con exactitud quién debe resolverlo & 0,283 & \\
\hline \multicolumn{3}{|l|}{ Administración del Talento Humano } \\
\hline La entidad me incentiva cuando alcanzo mis objetivos y metas & 0,663 & \\
\hline Generalmente la persona que se vincula a la entidad recibe entrenamiento adecuado para realizar su trabajo. & 0,639 & \\
\hline Estoy satisfecho con las actividades de bienestar que se realizan en la entidad & 0,549 & \\
\hline La entidad concede los encargos a las personas que los merecen. & 0,546 & \\
\hline Los empleados de la entidad ponen en práctica los valores institucionales & 0,484 & \\
\hline Existe un nivel adecuado de comunicación entre las diferentes áreas de la entidad. & 0,473 & \\
\hline La capacitación es una prioridad en la entidad & 0,47 & \\
\hline La entidad me tiene en cuenta para la planeación de sus actividades. & 0,466 & \\
\hline Me entero de lo que ocurre en mi entidad, más por comunicaciones oficiales que por comunicaciones informales & 0,451 & \\
\hline Cuento con los recursos necesarios para realizar mi trabajo eficientemente & 0,428 & 0,83 \\
\hline \multicolumn{3}{|l|}{ Trabajo en grupo } \\
\hline En mi área de trabajo se fomenta el trabajo en grupo. & 0,252 & \\
\hline Estoy satisfecho con la forma en que me comunico con mis compañeros & 0,705 & \\
\hline $\begin{array}{l}\text { En mi área de trabajo se ha logrado un nivel de comunicación con mis compañeros de trabajo que facilita el logro de } \\
\text { los resultados }\end{array}$ & 0,702 & \\
\hline Los objetivos de los grupos en los que yo he participado son compartidos por todos sus integrantes & 0,702 & \\
\hline Mi grupo trabaja con autonomía respecto de otros grupos & 0,623 & \\
\hline Los procedimientos de mi área de trabajo garantizan la efectividad de las acciones que se realizan & 0,558 & \\
\hline En mi área mantenemos las prioridades para el desarrollo del trabajo & 0,352 & \\
\hline $\begin{array}{l}\text { Considero que las personas de los grupos en que he participado, aportan lo que se espera de ellas para la realización } \\
\text { de las tareas }\end{array}$ & 0,32 & \\
\hline $\begin{array}{l}\text { Considero que las personas de los grupos en que he participado, aportan lo que se espera de ellas para la realización } \\
\text { de las tareas }\end{array}$ & 0,297 & 0,76 \\
\hline En mi área compartimos información útil para el trabajo & 0,291 & \\
\hline \multicolumn{3}{|l|}{ Medio ambiente físico } \\
\hline Mi área de trabajo permanece ordenada & 0,729 & \\
\hline Mi área de trabajo permanece limpia & 0,725 & 0,84 \\
\hline En mi área de trabajo se efectúan oportunamente las actividades de mantenimiento que sean necesarias & 0,602 & \\
\hline En mi área de trabajo la iluminación es la adecuada & 0,568 & \\
\hline Mi área de trabajo tiene suficiente ventilación & 0,559 & \\
\hline Mi área de trabajo tiene un ambiente físico seguro (ergonomía) & 0,551 & \\
\hline En mi área de trabajo no hay contaminación auditiva (ruido) & 0,525 & \\
\hline
\end{tabular}




\section{Resultados}

La población estuvo conformada por 410 funcionarios, de los cuales el $49,8 \%$ fueron mujeres; las edades más frecuentes oscilan entre 30 y 59 años (75\%), en un menor porcentaje se encuentran edades de los 18 a 29 años (17\%), y un 8\% se encontraron entre los 60 o más años. El estado civil más recurrente fue casado(a) (46\%) y soltero(a) (36\%).

\section{Clima organizacional}

Se obtuvo una medida de favorabilidad o desfavorabilidad de los servidores públicos frente a su entorno laboral, a partir de las respuestas dadas por los sujetos en los diferentes ítems. Se encontró de manera general que el $79 \%$ de los funcionarios perciben el clima organizacional como favorable.

En cuanto a las dimensiones que componen esta variable, se pueden percibir mayores niveles de favorabilidad en la capacidad profesional y estilo de dirección. Así mismo, se puede evidenciar que las únicas dimensiones que no superan el $75 \%$ de favorabilidad son administración del talento humano y medio ambiente (Figura 1).

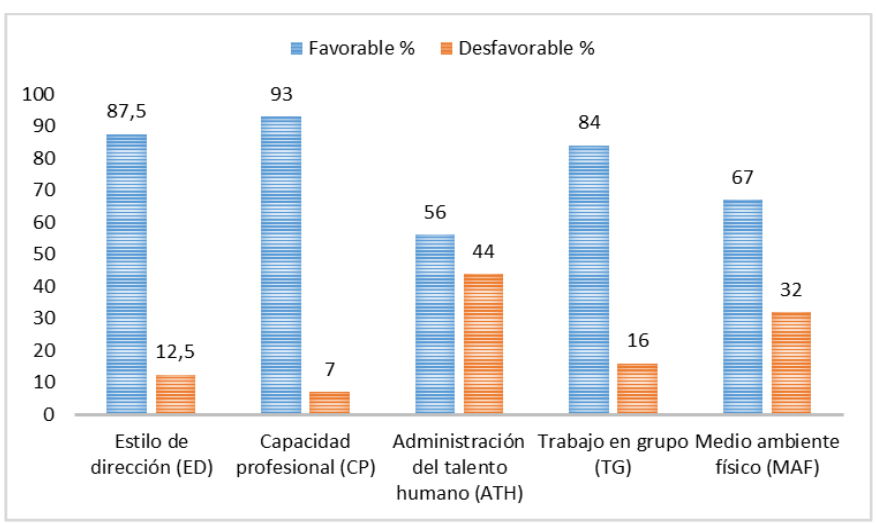

Figura 1. Favorabilidad vs. desfavorabilidad del clima organizacional por dimensiones
Teniendo en cuenta los cargos en los que se desempeñan los funcionarios se puede mencionar que administrativos, docentes y personal de apoyo perciben un nivel semejante de favorabilidad de clima organizacional, que oscila aproximadamente entre el $78 \%$ y $79 \%$. Por su parte, en los directivos el porcentaje de favorabilidad sobresale con un $84,8 \%$.

En referencia al nivel educativo de los participantes, no se presentaron diferencias significativas en cuanto a la percepción favorable del clima organizacional se encuentra un porcentaje de favorabilidad que oscila entre el $79 \%$ y el $80 \%$; en el caso puntual del funcionario sin ningún nivel de educación formal $(n=1)$ y los que cursaron como último nivel de estudio la primaria $(\mathrm{n}=14)$ presentaron un $82 \%$ de favorabilidad.

\section{Violencia laboral}

Se evaluó la presencia de violencia laboral considerándola alta, media o nula, en correspondencia a la cantidad de diferentes formas o eventos de violencia que la persona sufre en su ambiente de trabajo y se determina por el número de reactivos en que el sujeto contestó como positivo frente a la presencia de conductas violentas. En el nivel alto se ubican el 34\% de la población, un 39\% en un nivel medio y un $27 \%$ reportó que no había recibido ningún tipo de violencia en su ámbito laboral.

Teniendo en cuenta que el instrumento de medición permite obtener tanto valores de presencia de violencia laboral general, como valores independientes para cada uno de los factores (MIC, DIP, ODL) que componen esta variable. Cada uno de estos factores tiene una medida cuantitativa propia en la que los participantes han contestado la totalidad de los ítems (Tabla 2).

Tabla 2. Presencia de violencia laboral

\begin{tabular}{lccc}
\hline \multirow{2}{*}{ Niveles } & \multicolumn{2}{c}{ Factores de violencia laboral } \\
\cline { 2 - 4 } & $\begin{array}{c}\text { Manipulación para inducir al castigo } \\
\text { (MIC) \% }\end{array}$ & $\begin{array}{c}\text { Daños a la imagen pública (DIP) } \\
\text { \% }\end{array}$ & $\begin{array}{c}\text { Obstáculos al desempeño } \\
\text { laboral (ODL) \% }\end{array}$ \\
\hline Alto & 16 & 11 & 4 \\
Medio & 49 & 44 & 23 \\
Nulo & 35 & 45 & 73 \\
Total & 100 & 100 & 100 \\
\hline
\end{tabular}


Se observa que las puntuaciones más altas de los factores de violencia laboral evaluados se ubican en el nivel nulo y medio.

Por su parte, la intensidad de violencia laboral se cuantifica uniendo en número de conductas violentas presentes a la frecuencia con que cada una de estas conductas se da, de acuerdo a los resultados se encontró que el 10\% de la población se ubica en el nivel alto y medio.

\section{Acoso psicológico}

En cuanto al acoso psicológico en el trabajo se reportó un 10,2 \%. En el nivel alto se ubicó el 4,4\% y en el nivel medio el 5,8\%, el resto de la población se ubicó en los niveles bajo y nulo.

\section{Relación entre clima organizacional y violencia laboral}

Previo al análisis de la relación entre las dos variables, se aplicó la prueba de Kolmogorov-
Smirnov. Considerando la ausencia de normalidad que estos presentaron se aplicó el coeficiente de correlación de Spearman, obteniendo como resultado que, entre el clima organizacional y la violencia laboral existe una importante relación negativa $(r=-0,441, p<0,005)$, esto implica que la presencia de violencia se relaciona con bajos niveles o ausencia de favorabilidad de clima organizacional y viceversa (Tabla 3). Dentro de estas dimensiones de identifica una correlación negativa más estrecha entre la violencia laboral y el estilo de liderazgo (ED); este factor también presenta una alta correlación con el factor obstáculos al desempeño laboral (ODL) y este, a su vez se encuentra en un nivel semejante de correlación con respecto a administración del talento humano (ATH). La variable clima organizacional se correlacionó de manera importante con los factores de violencia laboral MIC Y ODL (Tabla 3).

Tabla 3. Correlación clima organizacional y violencia laboral

\begin{tabular}{|c|c|c|c|c|c|}
\hline & VL & APT & DIP & MIC & ODL \\
\hline ED & $-0,441^{* *}$ & $-0,347^{* *}$ & $-0,366^{* *}$ & $-0,376^{* *}$ & $-0,433^{* *}$ \\
\hline $\mathrm{CP}$ & $-0,304^{* *}$ & $-0,184^{* *}$ & $-0,190^{* *}$ & $-0,240^{* *}$ & $-0,336^{* *}$ \\
\hline ATH & $-0,424^{* *}$ & $-0,261^{* *}$ & $-0,271^{* *}$ & $-0,371^{* *}$ & $-0,406^{* *}$ \\
\hline TG & $-0,340^{* *}$ & $-0,292^{* *}$ & $-0,245^{* *}$ & $-0,323^{* *}$ & $-0,331^{* *}$ \\
\hline MAF & $-0,207^{* *}$ & $-0,197^{* *}$ & $-0,155^{* *}$ & $-0,197^{* *}$ & $-0,208^{* *}$ \\
\hline CL & $-0,474 * *$ & $-0,342^{* *}$ & $-0,348^{* *}$ & $-0,415^{* *}$ & $-0,462^{* *}$ \\
\hline
\end{tabular}

VL: Violencia laboral; APT: Acoso psicológico en el trabajo; DIP: Daños a la imagen pública MIC: Manipulación para inducir al castigo; ODL: Obstáculos al desempeño laboral; ED: Estilo de dirección; CP: Capacidad profesional; ATH: Administración del talento humano; TG: Trabajo en equipo; MAF: Medio ambiente físico; CL: Clima laboral. Se resalta en negrita el valor que representa la mayor correlación entre variables. Los asteriscos representan la significancia de la correlación $\left(p<0,05^{*}, p<0,01^{* *}\right)$

\section{Discusión}

Los resultados de la variable clima laboral, mostraron que los funcionarios en general reportaron una percepción favorable, de los cuales sobresale el grupo de directivos con un nivel de percepción de mayor favorabilidad, lo cual permite reafirmar que el tipo de cargo posiblemente está relacionado con la percepción del clima laboral(34). Sin embargo, es importante aclarar que la diferencia en la percepción entre directivos y el resto de funcionarios evaluados, no se relaciona con el nivel de educación, ya que no se encontraron diferencias estadísticamente significativas en cuanto a esta característica sociodemográfica.

De las dimensiones que componen el clima laboral se destaca la capacidad profesional (CP) con una alta favorabilidad. Estos resultados se enlazan con hallazgos previos en otras investigaciones(35,36), en los cuales se observa que la CP denota un panorama sobre la forma en que los colaboradores están utilizando sus competencias y desarrollando aún más sus habilidades, aspecto que aporta a la organización pero también al desarrollo del talento humano ,lo cual repercute directamente en su bienestar personal, profesional y laboral.

Por el contrario, la que presenta el menor porcentaje de favorabilidad es la administración del talento humano (ATH), dimensión que es necesario profundizar, ya que los procesos de selección, inducción, contratación, capacitación, evaluación del desempeño, seguridad y salud en el trabajo y bienestar podrían afectar de manera desfavorable a la percepción del clima organizacional. Esta situación ha sido encontrada por otros autores, quienes han reportado un descontento por parte de 
los colaboradores con relación a la oferta de oportunidades de realización personal y laboral en la organización(5,34-36), razón por la cual es recomendable que se dirija la atención hacia el análisis de esta variable, dado que el clima laboral tal como se administre en la institución representara el desarrollo integral y el bienestar de los funcionarios, las acciones orientadas a velar por el crecimiento personal y profesional que aportan a mejorar la satisfacción y la calidad de vida laboral de los funcionarios en la institución, lo que puede generar un mayor compromiso que a la innovación y desarrollo de la organizacion ${ }^{37)}$.

La administración integral del talento humano al servicio del Estado, es una condición necesaria para que los organismos públicos funcionen internamente y que el servicio se preste de manera adecuada, generando los resultados que espera la sociedad(11). En este aspecto se resalta el papel de los directivos en las áreas administrativas del ámbito público(38), quienes están en la obligación de brindar metodologías y capacitaciones a los funcionarios de una institución, para garantizar un buen clima organizacional y contribuir en la prestación de un mejor servicio a los usuarios ${ }^{(5)}$.

Se ha reconocido que dependiendo del tipo de liderazgo ejercido, puede emerger una variabilidad que va desde un clima favorable a uno desfavorable(38). Identificándolo como un factor que influye directamente en el clima laboral, debido a la relación recíproca existente entre liderazgo y clima organizacional en las instituciones educativas(39). Esto se asemeja a los hallazgos del presente estudio, ya que la puntuación de "liderazgo" es la segunda con mayor favorabilidad, permitiendo inferir que la dinámica de esta institución se desarrolla de manera que el estilo de dirección mejora la percepción que los colaboradores tienen con respecto al ambiente laboral.

Dada la favorabilidad de la dimensión trabajo en grupo (TG), se reafirma la estrecha asociación entre esta y el clima laboral ${ }^{(40)}$. El estado satisfactorio del TG evidencia la efectividad del desempeño de los colaboradores, mediante relaciones interpersonales adecuadas y de cooperación. De esta manera, se espera que las dinámicas entre los integrantes de esta institución, faciliten el logro de resultados y cumplimiento de metas.
Los resultados demuestran que en este momento el clima organizacional percibido es altamente favorable; sin embargo, se requiere realizar un continuo seguimiento, puesto que las organizaciones son dinámicas y existen múltiples factores externos e internos que se vivencian a diario $y$ pueden afectarlo(5).

En cuanto a la violencia laboral los resultados muestran situaciones que requieren una intervención, al considerar el porcentaje de población que manifestaron percibir un nivel alto o medio de violencia laboral; esta variable ocasiona diferentes tipos de conflictos relacionados con la disminución del rendimiento laboral, el bienestar del trabajador y afecta en gran medida a la organización(26). Tomando en cuenta los factores que componen esta variable (MIC, DIP, ODL) se infiere desde la definición operativa del instrumento aplicado y desde la Ley 1010 de 2006 de acoso laboral, que la mayoría de los actos violentos encontrados en esta Institución se enfocan probablemente en disponer situaciones con el objetivo de perjudicar al otro, de manera que se provoque injustamente el castigo o incluso el despido, afirmación que requiere de otros estudios para complementar su análisis.

Los comportamientos relacionados a violencia laboral de alta intensidad, presentan porcentajes bajos, es decir, los actos de violencia se manifiestan de manera aislada, sin perjudicar en mayor medida a la población. Es importante tener en cuenta que los porcentajes de los resultados de acoso psicológico en el trabajo en esta investigación (10,2\%), son relativamente similares respecto a los encontrados en México (8,5\%), utilizando el mismo instrumento; sin embargo, es necesario tener en cuenta que realizar comparaciones de resultados sobre la medición y variaciones en la prevalencia de la variable en diferentes países (México, Chile, España, Costa Rica, Bolivia, Ecuador y Cuba) dadas las posibles diferencias en la población (como por ejemplo con docentes universitarios), razones culturales y por los diferentes sentidos que se le asigna al atributo de acoso laboral y a las variadas estrategias para medirlo, resulta complicado(41). Por lo tanto, es fundamental incluir en los estudios sobre violencia laboral muestras del sector educativo, que brinden elementos de análisis y reflexión frente al tema de estudio, resaltando la importancia de poner 
en marcha un plan de acción para disminuir y controlar estos comportamientos hostiles.

Más aún, en algunos estudios se ha logrado determinar una relación entre acoso y clima laboral(2-5,28,29), articulándose con los resultados del presente estudio. En esta línea se ha determinado que la presencia de prácticas de acoso resulta contingente, tanto al tipo de clima que predomina en dicho entorno, como a los procesos de cambio organizacional que se dan en el mismo, de igual manera se afirma que un ambiente de trabajo caracterizado por el apoyo entre sus miembros, ejerce un efecto preventivo para las situaciones de $\operatorname{acoso}{ }^{(28)}$.

Con respecto al estilo de dirección se destaca la estrecha correlación negativa con la presencia de violencia laboral, de esta manera se observa que pese al bajo porcentaje de funcionarios que han sido víctimas de violencia laboral en cualquiera de sus formas o niveles, posiblemente estos tienen una perspectiva negativa de sus jefes y de los altos mandos de la institución, alterando la atmósfera organizacional. Igualmente, se resalta la importante correlación inversa entre la ATH y ODL, que permite mencionar que posiblemente la falta de oportunidades de crecimiento profesional y personal evidenciada en el porcentaje de desfavorabilidad de la ATH, se asocia con actos contemplados en la evaluación del factor OLD, en los que probablemente se obstaculiza el desempeño de la víctima, ocultando información, bloqueando o impidiendo oportunidades de mejora en el trabajo, aportando a la teoría de la bidireccionalidad(2). Por el contrario, el medio ambiente físico (MAF) es el que presenta menor coeficiente de correlación con la violencia laboral y el acoso psicológico en el trabajo y sus factores, dejando de manifiesto que los participantes no lo perciben como un factor de riesgo para la emergencia de comportamientos de violencia en el lugar de trabajo.

Con base en la literatura revisada, se puede destacar que a pesar de que existen estudios que confirman la relación entre el acoso psicológico en el trabajo y el clima laboral(2-5,28,29), es limitada la investigación que existe con respecto a esta relación con violencia laboral propiamente dicha; por ello, el principal aporte del presente estudio se ha focalizado en reconocer la prevalencia de la violencia y su relación con respecto al clima organizacional. Ante esta relación debería considerarse realizar una intervención dirigida a disminuir la presencia de violencia en el trabajo; esta sería efectiva para la prevención de prácticas de acoso laboral y sus devastadores efectos, y favorecería las percepciones del ambiente laboral(28,29). En este caso, es importante que las directivas de la institución reconozcan la relación evidenciada entre estas dos variables, con el fin de crear estrategias efectivas para gestionar tanto la violencia laboral como el acoso psicológico en el trabajo y con esto, observar el posible efecto en la estabilidad de un clima laboral favorable.

\section{Conclusiones}

En general, los funcionarios de la institución conocen e identifican su organización, así como el sentido de su labor, orientando sus acciones hacia el cumplimiento de los objetivos institucionales. Se acepta la hipótesis de investigación, concluyendo que a mayor favorabilidad de clima organizacional menor es la presencia de acoso laboral.

Los comportamientos característicos de obstáculos al desempeño laboral y los referentes a manipulación para inducir al castigo son los que se asocian en mayor medida con la desfavorabilidad del clima organizacional.

El medio ambiente físico se reconoció como una dimensión del clima laboral que no se asocia de manera importante con la incidencia de violencia laboral en la institución de educación superior, infiriendo que la violencia laboral está más asociada con las interacciones entre los funcionarios de la institución, que con las condiciones físicas que rodean al trabajador.

La presente investigación implica el acercamiento y reconocimiento de la violencia laboral como una variable determinante en la incidencia de casos de hostigamiento en el lugar de trabajo, así como la desfavorabilidad de la percepción del ambiente laboral evidenciada en la correlación inversa comprobada en este estudio. En este sentido se sugiere continuar con esta línea de investigación, con el fin de profundizar en la relación del clima organizacional con la base del acoso psicológico en el trabajo, que como se refiere en esta investigación es la violencia laboral. 
Conflictos de interés: Los autores declaran que no existen conflictos de intereses.

\section{Referencias}

1. Garbanzo-Vargas GM. Desarrollo organizacional y los procesos de cambio en las instituciones educativas, un reto de la gestión de la educación. Revista Educación [Internet]. or2016;40(1):67-87. Recuperado de: http://www.redalyc.org/articulo.oa?id=44043204005

2. Ramos J. Relación entre Acoso laboral y clima organizacional: Revisión Teórica. [Tesis]. Cundinamarca: Universidad de la sabana; 2010. Disponible en https://intellectum.unisabana.edu.co/bitstream/handle/10 818/7517/124027.pdf?sequence=1\&isAllowed=y

3. Izcurdia M. Clima laboral y mobbing en el noveno encuentro de investigadores en psicología del Mercosur. Buenos Aires, Argentina: $V$ Congreso Internacional de Investigación y Práctica Profesional en Psicología XX Jornadas de Investigación; 2013. Disponible en https://www.aacademica.org/000-054/527.pdf

4. Qureshi M, Rasli A, Zaman A. A new trilogy to understand the relationship among organizational climate, workplace bullying and employee health. Arab economics and business journal. 2014;9(1):133-146. Disponible en https://www.sciencedirect.com/science/article/pii/S2214 462514000176

5. Ribera G, Briceño L. Impacto del acoso laboral vs Clima organizacional en las entidades públicas "el silencio de los servidores públicos". [Tesis]. Bucaramanga: Universidad Abierta y a Distancia UNAD; 2015. Disponible en https://repository.unad.edu.co/retrieve/4515/63325453.p df

6. Flores-Ortiz M, Vega-López A, Chávez-Moreno E. El clima organizacional como factor de competitividad en las franquicias de comida rápida en Tijuana, B.C., México. Revista Internacional Administración \& Finanzas. 2015;8(5):25-44. Disponible en https://www.researchgate.net/publication/275523192_EL_ CLIMA_ORGANIZACIONAL_COMO_FACTOR_DE_COMPETITI VIDAD_EN_LAS_FRANQUICIAS_DE_COMIDA_RAPIDA_EN_TIJ UANA_BC_MEXICO

7. Bernal I, Pedraza N, Sánchez M. El clima organizacional y su relación con la calidad de los servicios públicos de salud: diseño de un modelo teórico. Estudios Gerenciales. 2015;31(134):8-19. Disponible en https://ac.elscdn.com/S0123592314001624/1-s2.0-

S0123592314001624-main.pdf?_tid=e8396329-3095-4704acef-

40f9bd23f5ba\&acdnat $=1551709014 \_0 a 1621$ c8ec0621c5a1 6a022120dde4fa

8. Bolaños K, Franco D. Clima Organizacional e Identificación Organizacional en Empleados de una Empresa de la Ciudad de Cali. [Tesis]. [Cali]: Pontificia Universidad Javeriana; 2017. Disponible http://vitela.javerianacali.edu.co/bitstream/handle/11522 /8406/Clima_organizacional_identificacion.pdf?sequence $=1$ \&isAllowed=y

9. Viloria J, Pertúz S, Daza A, Pedraza L. Aproximación al clima organizacional de una empresa promotora de salud. Revista Cubana de Salud Pública. 2016;42(1):80-91. Disponible en https://www.scielosp.org/article/ssm/content/raw/?resou rce_ssm_path=/media/assets/rcsp/v42n1/spu09116.pdf

10. Bermúdez J, Pedraza A, Rincón C. El clima organizacional en universidades de Bogotá desde la perspectiva de los estudiantes. Revista electrónica de investigación educativa. 2015;17(3):01-12. Disponible en https://redie.uabc.mx/redie/article/view/413/12

11. Departamento Administrativo de la Función Pública. Guía de intervención para la Cultura organizacional, el Clima Laboral y el Cambio Organizacional.Bogotá: Departamento Administrativo de la Función Pública; 2005. Disponible en http://cdim.esap.edu.co/BancoMedios/Documentos\%20PD F/guiaclimaculturacambio.pdf

12. Charry H. La gestión de la comunicación interna y el clima organizacional en el sector público; Comuni@cción. 2018;9(1):25-34. Disponible en http://www.scielo.org.pe/scielo.php?script=sci_arttext\&pid =S2219-71682018000100003\&lng=es\&tlng=es

13. Riquelme A. Mobbing, un tipo de violencia en el lugar de trabajo. Revista electrónica de ciencias sociales online. 2006;3(2):39-57. Disponible http://docplayer.es/2893644-Mobbing-un-tipo-deviolencia-en-el-lugar-de-trabajo.html

14. Toro J, Gómez-Rubio C. Factores Facilitadores de la Violencia Laboral: Una Revisión de la Evidencia Científica en América Latina. Ciencia y Trabajo. 2018;56:110-116. Disponible https://scielo.conicyt.cl/pdf/cyt/v18n56/art06.pdf

15. Tuya-Figueroa X, Mezones-Holguin E, Monge E, Arones R, Mier M, Sarama M, Torres J, Mayta-Tristan P. Violencia laboral externa tipo amenaza contra médicos en servicios hospitalarios de Lima metropolitana, Perú 2014. Rev Peru Med Exp Salud Pública. 2016;33(4):670-9. doi: 10.17843/rpmesp.2016.334.2550

16. Galian I, Llor P, Ruiz J, Jiménez J. Exposición a violencia de los usuarios y satisfacción laboral en el personal de enfermería de los hospitales públicos de la región de Murcia. An. Sist. Sanit. Navar. 2018;41(2):181-189. Disponible en https://recyt.fecyt.es/index.php/ASSN/article/view/63846 /40702

17. Palma A, Ahumada M, Ansoleaga E. ¿Cómo afrontan la violencia laboral los trabajadores/as chilenos/as?. Psicoperspectivas, individuo y sociedad. 2018;17(3):1-13. Disponible http://www psicoperspectivas.cl/indexphp/psicoperspecti vas/article/view/1288/949

18. Porras N. Violencia psicológica en el trabajo: Aproximaciones desde la perspectiva psicosocial. Revista de la Asociación Latinoamericana para la Formación y Enseñanza de la Psicología. 2017;5(13):20-27. Disponible en http://integracion-

academica.org/attachments/article/150/02\%20Violencia\% 20psicologica\%20trabajo\%20-\%20NRPorras.pdf

19. Gómez M. Violencia en el puesto de trabajo sanitario, análisis situacional y experiencias de las víctimas. [Tesis]. España: Universidad Rovira i Virgili; 2015. Disponible en https://www.tesisenred.net/bitstream/handle/10803/401 582/TESIS_pdf?sequence $=2 \&$ isAllowed $=y$

20. Parra L, Acosta M. La investigación cuantitativa del acoso psicológico laboral en los sectores de la educación superior y de la salud. Una revisión sistemática. Entramado. 2010;6(1):158-172. Disponible http://www.redalyc.org/articulo.oa?id=265419646010 
21. Zambrano C, Perugache A, Figueroa J. Manifestaciones de la violencia basada en género en docentes universitarios. Psicogente. 2017;20(37):146-15. Disponible en http://www.scielo.org.co/pdf/psico/v20n37/0124-0137psico-20-37-00147.pdf

22. Leymann H. Important note in preface to Heinz Leymann, "Mobbing and Psychological Terror at Workplaces." Violence and Victims. 1990;5(2):119-126. Disponible en http://www.mobbingportal.com/LeymannV\&V1990(3).pdf

23. Escartín J, Monzani L, Leong F, Rodriguez A. A reduced form of the Workplace Bullying Scale the EAPA-T-R: A useful instrument for daily diary and experience sampling studies. WORK \& STRESS. 2017;13(1):42-62. Disponible en https://www.tandfonline.com/doi/abs/10.1080/02678373 .2017.1295113?journalCode=twst20

24. Villagrán S, Jasso D, Aldaba M, Rodríguez M. Mobbing: Impacto psicológico en docentes universitarios, repercusiones en el sentido de pertenencia y permanencia laboral. Pensamiento Americano. 2016;10(18):77-95. doi: doi.org/10.1080/02678373.2017.1295113

25. Congreso de Colombia. Ley 1010 de 2006. Disponible en http://www.secretariasenado.gov.co/senado/basedoc/ley_ 1010_2006.html

26. Pando M, Aranda C, Parra L, Gutiérrez A. Determinación del mobbing y validación del Inventario de Violencia y Acoso Psicológico en el Trabajo (IVAPT) para Colombia. Salud Uninorte. 2013;29(3):525-533. Disponible en http://rcientificas.uninorte.edu.co/index.php/salud/article /view/5171/3559

27. Ministerio de Salud y Protección Social. Formas y consecuencias de la violencia laboral. Colombia. Bogotá: MinSalud; 2004. Disponible en http://fondoriesgoslaborales.gov.co/documents/publicacio nes/estudios/Estudio-violencia-en-el-trabajo-2004.pdf

28. Muñoz H, Guerra J, Barón M, Munduate L. El acoso psicológico desde una perspectiva organizacional. Papel del clima organizacional y los procesos de cambio. Revista de Psicología del Trabajo y de las Organizaciones. 2006;2(3):347-361. Disponible

en https://idus.us.es/xmlui/bitstream/handle/11441/23220/ file_1.pdf;sequence $=1$

29. Chullo A, Taco E. Clima laboral y acoso laboral en los trabajadores de una cadena de pizzerías [Tesis]. Arequipa: Universidad Nacional de San Agustín de Arequipa; 2016. Disponible http://repositorio.unsa.edu.pe/handle/UNSA/3639

30. Hernández R, Fernández $\mathrm{C}$, Baptista P. Metodología de la investigación. 6ta Ed. México: MacGraw Hill; 2010.

31. Abad F, Olea J, Ponsoda V, García C. Medición en ciencias sociales y de la salud. España: Sintesis; 2011.

32. Segura S, Ferreres-Traver A, Hernández-Baeza A, TomásMarco I. El análisis factorial exploratorio de los ítems: una guía práctica, revisada y actualizada. Anales de Psicología. 2014;30(3):1151-1169.

Disponible http://scielo.isciii.es/pdf/ap/v30n3/metodologia1.pdf
33. Ministerio de Salud y Protección Social. Resolución 8430 de 1993. Bogotá: MinSalud; 1993. Disponible en https://www.minsalud.gov.co/sites/rid/Lists/BibliotecaDig ital/RIDE/DE/DIJ/RESOLUCION-8430-DE-1993.PDF

34. Williams, L. Estudio diagnóstico de clima laboral en una dependencia pública. [Tesis]. Monterrey: Universidad Autónoma de Nuevo León; 2013. Disponible en http://eprints.uanl.mx/3751/1/1080256607.pdf?fbclid=Iw AR1PwRQZ_aElZGJVL78rZToHkFCgUPZNOaN4GdA5kfju8j6 c09-VXijj05w

35. Cardona K. La satisfacción laboral y el clima organizacional en el CDI San José de la ciudad de Manizales. [Tesis]. Manizales: Universidad de Manizales; 2016. Disponible en http://ridum.umanizales.edu.co:8080/xmlui/handle/6789/ 3033

36. Polentino M, Reza D. Diagnóstico del clima laboral de los colaboradores de la planta de administrativos de la alcaldía del municipio de Villavicencio. [Tesis]. Acacias: Universidad Nacional Abierta y a Distancia UNAD; 2016. Disponible en http://stadium.unad.edu.co/preview/UNAD.php?url=/bitst ream/10596/9160/1/30080442.pdf

37. Pedraza N, Bernal I. El clima organizacional en el sector público y empresarial desde la percepción del capital humano. Revista Lasallista de Investigación. 2018;39(13):16-30. Disponible en https://www.revistaespacios.com/a18v39n13/a18v39n13 p16.pdf

38. Pecino V, Mañas M, Díaz P, López J, Llopis J. Clima y satisfacción laboral en el contexto universitario. Anales de Psicología. 2015;31(2):658-666. Disponible en http://scielo.isciii.es/scielo.php?script=sci_arttext\&pid=S02 12-97282015000200030

39. Contreras D, Jiménez L. Liderazgo y Clima Organizacional en un colegio de Cundinamarca. Lineamientos básicos para su intervención. [Tesis]. Bogotá: Universidad del Rosario; $2016 . \quad$ Disponible en http://repository.urosario.edu.co/bitstream/handle/10336 /11983/52982530-2016.pdf?sequence=9

40. Gonzales D, Ospina Y. Trabajo en equipo en el clima organizacional de las empresas, en el área específica del talento humano. Colección académica de Ciencias Sociales. 2015;2(1):119-142. Disponible en https://revistas.upb.edu.co/index.php/cienciassociales/arti cle/view/4326

41. Pando M, Aranda C, Preciado L, Franco S, Salazar J. Validez y confiabilidad del Inventario de Violencia y Acoso Psicológico en el Trabajo (IVAPT-PANDO). Enseñanza e Investigación en Psicología. 2006;11(2):319-332. Disponible en https://www.redalyc.org/articulo.oa?id=29211208 